Pacific Journal of Mathematic 


\title{
THE SCHUR INDEX FOR PROJECTIVE REPRESENTATIONS OF FINITE GROUPS
}

\author{
BURTON FEIN
}

In this paper the question of determining the absolutely irreducible constituents of an irreducible projective representation of a finite group is considered from the viewpoint of the theory of algebras. New proofs are given for several of the main results of the theory of representations of finite dimensional associative algebras. This theory is applied to determine the center of a simple component of a twisted group algebra modulo its radical and sufficient conditions are given to insure that this center is a normal extention of the base field. The Schur index of an absolutely irreducible projective representation of a finite group is defined as in the theory of linear representations of finite groups. It is shown that every irreducible complex projective representation of a finite group is projectively equivalent to a representation whose associated factor system has values which are roots of unity but whose Schur index over the rationals is 1 .

Throughout this paper all modules will be assumed to be finite dimensional unitary left modules. By an algebra over a field $K$ we shall mean a finite dimensional associative algebra in which the identity of $K$ acts as identity. If $\mathfrak{A}$ is an algebra, we denote the radical of $\mathfrak{A}$ by $\operatorname{rad} \mathfrak{A}$ and set $\overline{\mathfrak{A}}=\mathfrak{A} / \mathrm{rad} \mathfrak{A}$. If $E$ is an extension field of $K$, we identify $\mathfrak{A}$ as a subalgebra of $\mathfrak{A}^{E}=E \bigotimes_{K} \mathfrak{A}$. If $M$ is an $\mathfrak{A}$-module, we denote by $M^{E}$ the $\mathfrak{A}^{E}$-module, $E \otimes_{K} M$. We refer the reader to [4] for the relevant theory of algebras assumed.

1. The Schur index. Let $\mathfrak{U}$ be an algebra over the perfect field $K$. There exists a finite normal (separable) extension field $E$ of $K$ which is a splitting field for $\mathfrak{A}$, i.e., $\mathfrak{A}^{E} / \operatorname{rad}\left(\mathfrak{A}^{E}\right)$ is a direct sum of complete matrix rings over $E$. Let $\left\{x_{1}, \cdots, x_{s}\right\}$ be a basis for $\mathfrak{A}$ over $K$. Under the usual identification, $\left\{x_{1}, \cdots, x_{s}\right\}$ is also a basis for $\mathfrak{A}^{E}$. We denote the Galois group of $E$ over $K$ by $\$(E \mid K)$. Let $N$ be a left $\mathfrak{A}^{E}$-module with basis $\left\{m_{1}, \cdots, m_{n}\right\}$ over $E$ and let the module action be given by $x_{k} m_{i}=\sum_{j} a_{i j}\left(x_{k}\right) m_{j}, k=1, \cdots, s, a_{i j}\left(x_{k}\right) \in E$. Let $V$ be an $n$-dimensional vector space over $E$ with basis $\left\{v_{1}, \cdots, v_{n}\right\}$ and let $\sigma \in \mathbb{S}(E \mid K)$. Under the action $x_{k} v_{i}=\sum_{j} \sigma\left(a_{i j}\left(x_{k}\right)\right) v_{j}, k=1, \cdots, s, V$ becomes an $\mathfrak{A}^{E}$-module which we denote by $\sigma N$. $\sigma N$ is called a conjugate module to $N$ and the representations of $\mathfrak{A}^{E}$ that these modules afford are said to be conjugate (with respect to the pair of bases $\left.\left\{x_{1}, \cdots, x_{s}\right\},\left\{v_{1}, \cdots, v_{s}\right\}\right)$. By the character afforded by a representation 
$T$ of a $K$-algebra $\mathfrak{B}$, we shall mean the function $\mu$ from $\mathfrak{B}$ to $K$ defined by $\mu(x)=$ trace $(T(x)), x \in \mathfrak{B}$. If $\chi$ is the character of $\mathfrak{H}^{E}$ afforded by $N$, we denote by $\sigma \chi$ the character of $\mathfrak{U}^{E}$ afforded by $\sigma N$ (where $\left.\sigma \chi\left(x_{k}\right)=\sigma\left(\chi\left(x_{k}\right)\right), k=1, \cdots, s\right) . \quad \sigma$ and $\tau$ will always denote elements of $\mathbb{S}(E \mid K)$ while $\chi$ and $\psi$ will always be characters. $i, j$, $k, n, m$, etc., will always denote natural numbers.

Let $E^{*}$ be an algebraic closure of $E$. All fields considered will be assumed to be subfields of $E^{*}$. Let $M$ be an irreducible $\mathfrak{X}$-module. $M$ is isomorphic to a minimal left ideal of a simple component $\mathfrak{B}$ of $\overline{\mathfrak{A}}$. $\mathfrak{B}$ is isomorphic to a complete matrix ring $(D)_{t}, D$ a division algebra with center $L, L \supset K$. If $r$ is a natural number, $r M$ is defined to be the direct sum of $r$ copies of $M$. $N$ will be assumed to be an irreducible $\mathfrak{A}^{E}$-module which is a composition factor of $M^{E}$. $\chi$ will denote the character of $\mathfrak{U}^{E}$ afforded by $N . \quad K(\chi)$ will denote the field generated over $K$ by the values $\chi\left(x_{k}\right), k=1, \cdots, s$. Let $N^{*}=E^{*} \otimes_{K} N$. We say that $N^{*}$ is realizable in a subfield $F$ of $E^{*}, F \supset K$, if there is an $\mathfrak{A}^{F}$-module $W$ such that $E^{*} \bigotimes_{K} W \cong N^{*}$. If $N^{*}$ is realizable in a subfield $F$ of $E^{*}, F \supset K$, then clearly $F \supset K(\chi)$. We define the Schur index, $m_{K}(N)$, of $N$ with respect to $K$ to be the minimum of $\left(F: K(\chi)\right.$ ) taken over all extension fields $F$ of $K(\chi)$ in which $N^{*}$ is realizable. $m(D)$ will denote the index of $D$.

In this section we shall give new proofs of the following two results.

THEOREM 1.1. The center $L$ of $D$ is isomorphic to $K(\chi)$.

THEOREM 1.2. (a) $M^{E} \cong m_{K}(N)\left(\sigma_{1} N \oplus \cdots \oplus \sigma_{t} N\right)$ where $\sigma_{i} \in \mathbb{S}(E \mid K)$, $\sigma_{1}=1$, the $\left\{\sigma_{i} N\right\}$ form a complete set of non-isomorphic conjugates of the irreducible $\mathfrak{A}^{E}$-module $N$, and $t=(K(\chi): K)$.

(b) $m_{K}(N)=m(D)$.

(c) If $K$ is a finite field, $m_{K}(N)=1$.

(d) $m_{K}(N)$ divides $(N: E)$.

(e) $m_{K}(N)$ divides $(J: K(\chi))$, where $J$ is any finite algebraic extension of $K$ in which $N^{*}$ is realizable.

(f) If $m N$ is realizable in $K(\chi)$, then $m_{K}(N)$ divides $m$.

( $\mathrm{g})$ If $K$ is a finite field or has characteristic zero, then the characters of the nonisomorphic irreducible $\mathfrak{X}$-modules are linearly independent.

Theorem 1.1 is Satz 3 of [1]. Parts of Theorem 1.2 appear in [1], [2], and [10]. For the case when $\mathfrak{A}$ is the group algebra of a finite group over a field of characteristic zero, several treatments of these theorems have been given. References to the literature in this 
regard may be found in [11]. In a previous paper we presented a treatment of Theorems 1.1 and 1.2 for the case when $\mathfrak{A}$ was the group algebra of a finite group over an arbitrary field [5]. The proof that we gave for this special case can be modified to yield new proofs of Theorem 1.1 and Theorem 1.2 when $\mathfrak{A}$ is an arbitrary algebra over a perfect field.

Proof of Theorem 1.1. Let $L$ be the center of $D$. Extending $E$ if necessary, we may assume that $L$ is embedded in $E$. Since $N$ is absolutely irreducible we note that the proofs of Theorems 27.8, $30.12(\mathrm{i})$, and 30.15 of [4] are valid in our context. Thus $\sigma N \cong N$ if and only if $\sigma \chi=\chi$. From the proof of Lemma 1.2 of [5] (see also $[4$, p. 472]) we see, in particular, that the number $t$ of simple components of $\mathfrak{B}^{E}$ satisfies:

$$
t=(K(\chi): K)
$$

Since $K$ is perfect, $K(\chi)$ and $L$ are both separable extensions of $K$, so $\operatorname{rad}\left(\mathfrak{H}^{L}\right) \cong L \otimes \operatorname{rad} \mathfrak{A}\left[4\right.$, Th. 69.10]. Thus $\mathfrak{B}^{L}$ is a component of $\mathfrak{U}^{L} / \mathrm{rad} \mathfrak{U}^{L}$. The center of $\mathfrak{B}^{L}$ is isomorphic to $L \bigotimes_{K} L$ [7, Th. 1, p. 114]. However, $L \otimes_{K} L$ is the direct sum of all inequivalent $K$-composites of $L$ with itself [8, Th. 21, p. 84]. One of these composites is isomorphic to $L$ and so some simple component, $\sqrt{ }$, of $\mathfrak{B}^{L}$ is central simple. Let $W$ be the irreducible $\mathfrak{U}^{L}$-module afforded by a minimal left ideal of $\mathfrak{C}^{5} . \mathfrak{S}^{E}$ is a central simple component of $\mathfrak{A}^{E} / \mathrm{rad}\left(\mathfrak{U}^{E}\right)$ and without loss of generality we may assume that $N$ is afforded by a minimal left ideal of $\mathbb{C}^{E}$. Since $\mathbb{C}^{E}$ has only one simple component, we have by (1) that $L(\chi) \cong L$ and so $K(\chi)$ is embedded in $L$. Similarly, the center of $K(\chi) \bigotimes_{K} \mathfrak{B}$ is isomorphic to $K(\chi) \otimes_{K} L$. Let $\mathfrak{D}$ be the simple component of $K(\chi) \otimes_{K} \mathfrak{B}$ such that $N$ is afforded by a minimal left ideal of $\mathfrak{D}^{E}$. By (1) $\mathfrak{D}^{E}$ is central simple and so $\mathfrak{D}$ is central simple. Since the center of $\mathfrak{D}$ is also a composite of $K(\chi)$ and $L$ over $K$, we see that $K(\chi)$ contains a subfield isomorphic to $L$. Thus $L \cong K(\chi)$.

With the obvious modifications, the proofs of Lemma 1.3 and Theorem 1.4 of [5] prove Theorem 1.2.

2. Outer tensor products. Let $\mathfrak{A}_{1}$ and $\mathfrak{A}_{2}$ be algebras over an arbitrary field $K$ and let $\mathfrak{A}=\mathfrak{A}_{1} \otimes_{K} \mathfrak{A}_{2}$. Let $M_{i}$ be an irreducible $\mathfrak{N}_{i}$-module, $i=1,2$. The outer tensor product $M_{1} \# M_{2}$ of $M_{1}$ and $M_{2}$ is the $\mathfrak{A}$-module whose underlying space is $M_{1} \otimes_{K} M_{2}$ and where the module action is given by

$$
\left(a_{1} \otimes a_{2}\right) \sum m_{j} \otimes m_{j}^{\prime}=\sum a_{1} m_{j} \otimes a_{2} m_{j}^{\prime}
$$


where $m_{j} \in M_{1}, m_{j}^{\prime} \in M_{2}$, and $a_{i} \in \mathfrak{A}_{i}, i=1,2$.

In $[5, \S 2]$ the theory of the Schur index was applied to study the structure of $M_{1} \# M_{2}$ for the case when $\mathfrak{A}_{1}$ and $\mathfrak{A}_{2}$ were group algebras of finite groups over an arbitrary field. Although we are not primarily interested in studying the structure of $M_{1} \# M_{2}$ when $\mathfrak{A}_{1}$ and $\mathfrak{A}_{2}$ are twisted group algebras, we will show that the results obtained in $[5, \S 2]$ are valid for arbitrary finite dimensional algebras. Many of these results seem to be well known.

Proposition 2.1. If $M_{i}$ is an absolutely irreducible $\mathfrak{A}_{i}$-module, $i=1,2$, then $M_{1} \# M_{2}$ is an absolutely irreducible $\mathfrak{A}$-module.

Proof. Let $M_{i}$ afford the matrix representation $T_{i}$ of $\mathfrak{A}_{i} i=1,2$. Then $M_{1} \# M_{2}$ affords the matrix representation $S$ of $\mathfrak{A}$ where

$$
S\left(a_{1} \otimes a_{2}\right)=T_{1}\left(a_{1}\right) \times T_{2}\left(a_{2}\right)
$$

(× denoting Kronecker product), $a_{i} \in \mathfrak{A}_{i}$. If $T_{1}, T_{2}$ have degrees $r, s$, respectively, then $S$ has degree $r s$. It is sufficient to prove that $S(\mathfrak{U})=K_{r s}$. By assumption $T_{1}\left(\mathfrak{A}_{1}\right)=K_{r}, T_{2}\left(\mathfrak{A}_{2}\right)=K_{s}$. Let $Z$ be a matrix in $K_{r s}$. Since $K_{r} \otimes_{K} K_{s} \cong K_{r s}, Z=\sum_{i} A_{i} \times B_{i}, A_{i} \in K_{r}, B_{i} \in K_{s}$. Then $A_{i}=T_{1}\left(a_{i}\right), B_{i}=T_{2}\left(b_{i}\right), a_{i} \in \mathfrak{A}_{1}, b_{i} \in \mathfrak{A}_{2}$. Thus

$$
Z=\sum_{i} T_{1}\left(a_{i}\right) \times T_{2}\left(b_{i}\right)=\sum_{i} S\left(a_{i} \otimes b_{i}\right) \in S(\mathfrak{A}) \text {. }
$$

Throughout this section $K$ will denote an arbitrary field and $\mathfrak{A}_{1}$ and $\mathfrak{A}_{2}$ will be algebras over $K$ such that the centers of $\overline{\mathfrak{A}}_{1}$ and $\overline{\mathfrak{A}}_{2}$ are separable over $K$. All fields considered will be assumed to be subfields of a fixed algebraic closure of $E, E$ being a splitting field for $\mathfrak{A}, \mathfrak{A}_{1}$ and $\mathfrak{A}_{2} . \quad M_{1}$ and $M_{2}$ will denote irreducible $\mathfrak{A}_{1}$ and $\mathfrak{A}_{2}$-modules, respectively. We wish to investigate the case when one or both of the $M_{i}$ are not absolutely irreducible. The first part of the proof of Theorem 2.2 of [5] generalizes without difficulty to yield the following result.

Proposition 2.2. If the centers of $\overline{\mathfrak{A}}_{1}$ and $\overline{\mathfrak{A}}_{2}$ are separable over $K$, then $\overline{\mathfrak{A}}_{1} \otimes_{K} \overline{\mathfrak{A}}_{2} \cong \overline{\mathfrak{A}}$ and

$$
\operatorname{rad} \mathfrak{U}=\operatorname{rad} \mathfrak{A}_{1} \otimes \mathfrak{U}_{2}+\mathfrak{N}_{1} \otimes \operatorname{rad} \mathfrak{U}_{2} .
$$

When $K$ has characteristic zero, this result was proved by Ivan [6]. The result is false without the separability condition. For a general discussion of the behavior of the radical under tensor products, see [7, Chapter 5, §14].

As an immediate corollary we have: 
CoROLLARY 2.3. If the centers of $\overline{\mathfrak{A}}_{1}$ and $\overline{\mathfrak{A}}_{2}$ are separable over $K$, then $M_{1} \# M_{2}$ is completely reducible.

If $\mathfrak{A}_{1}$ and $\mathfrak{A}_{2}$ are group algebras, then Theorem 2.2 of [5] shows that $M_{1} \# M_{2}$ is the direct sum of irreducible 2 -modules of the same dimension with each isomorphism type appearing with the same multiplicity. As we shall see in $\S 3$ this result is false even for twisted group algebras over a field of characteristic zero. This result is true, however, if the centers of $\overline{\mathfrak{A}}_{1}$ and $\overline{\mathfrak{A}}_{2}$ are separable over $K$ and the center of the simple component of $\overline{\mathfrak{A}}_{i}$ affording $M_{i}$ is a normal extension of $K, i=1,2$. In view of the separability condition, in proving this result we may assume that $\mathfrak{A}_{1}$ and $\mathfrak{A}_{2}$ are simple algebras.

THEOREM 2.4. Assume that $\mathfrak{A}_{1}$ and $\mathfrak{A}_{2}$ are simple algebras over the arbitrary field $K$ and let $L_{i}$ be the center of $\mathfrak{A}_{i}, i=1,2$. If $L_{i}$ is a separable normal extension of $K, i=1,2$, then $M_{1} \# M_{2}$ is the direct sum of irreducible $\mathfrak{2}$-modules of the same dimension with each isomorphism type appearing with the same multiplicity.

Proof. Since $L_{1}$ and $L_{2}$ are separable normal extensions of $K$, $L_{1} \otimes_{K} L_{2}$ is isomorphic to a sum of copies of $L$, where $L=L_{1} L_{2}$ is the composite of $L_{1}$ and $L_{2}$ [8, Th. 21, p. 84]. Since the center of $\mathfrak{A}_{1} \bigotimes_{K} \mathfrak{A}_{2}$ is isomorphic to $L_{1} \bigotimes_{K} L_{2}$ we see that

$$
\mathfrak{A}_{1} \otimes \mathfrak{A}_{2} \cong \mathfrak{B}_{1} \oplus \cdots \oplus \mathfrak{B}_{r},
$$

where the $\mathfrak{B}_{i}$ are simple algebras over $K$, each having center $L$. We have

$$
\begin{aligned}
\mathfrak{B}_{i} \bigotimes_{K} L & \cong\left(\mathfrak{B}_{i} \bigotimes_{L} L\right) \bigotimes_{K} L \cong \mathfrak{B}_{i} \bigotimes_{L}\left(L \bigotimes_{K} L\right) \\
& \cong \mathfrak{B}_{i} \bigotimes_{L}(L \oplus \cdots \oplus L) \cong \mathfrak{B}_{i} \oplus \mathfrak{B}_{i} \oplus \cdots \oplus \mathfrak{B}_{i},
\end{aligned}
$$

where $\mathfrak{B}_{i}$ is viewed on the right hand side of this equation as an algebra over $L$. Therefore $\mathfrak{U} \otimes_{K} L$ is isomorphic to a sum of copies of the $\mathfrak{B}_{j}, j=1, \cdots, r$. However,

$$
\mathfrak{U} \bigotimes_{K} L \cong\left(\mathfrak{A}_{1} \bigotimes_{K} L\right) \bigotimes_{L}\left(\mathfrak{A}_{2} \bigotimes_{K} L\right)
$$

and

$$
\begin{aligned}
\mathfrak{A}_{i} \bigotimes_{K} L & \cong\left(\mathfrak{A}_{i} \otimes_{L_{i}} L_{i}\right) \bigotimes_{K} L \cong \mathfrak{A}_{i} \bigotimes_{L_{i}}\left(L_{i} \bigotimes_{K} L\right) \\
& \cong \mathfrak{A}_{i} \otimes_{L_{i}}(L \oplus \cdots \oplus L) \cong\left(\mathfrak{A}_{i} \otimes_{L_{i}} L\right) \oplus \cdots \oplus\left(\mathfrak{A}_{i} \otimes_{L_{i}} L\right) .
\end{aligned}
$$

Since $\mathfrak{A}_{i} \otimes_{L_{i}} L$ is central simple, we see that the $\mathfrak{B}_{i}$ are isomorphic simple algebras over $K$. Let $\mathfrak{B}_{i} \cong(\mathfrak{D})_{t}$, where $\mathfrak{D}$ is a division algebra with center $L$. Let $\mathfrak{F}_{i}$ be a minimal left ideal of $\mathfrak{U}_{i}, i=1,2$. Then 
$\mathfrak{U}=\mathfrak{A}_{1} \bigotimes_{K} \mathfrak{A}_{2}$ is isomorphic to a direct sum of copies of $\mathfrak{V}_{1} \bigotimes_{K} \mathfrak{V}_{2}$. As an $\mathfrak{A}_{i}$-module, $M_{i}$ is isomorphic to $\mathfrak{F}_{i}, i=1,2$, so as an $\mathfrak{Y}$-module, $\mathfrak{A}$ is isomorphic to a sum of copies of $M_{1} \# M_{2}$. Let $N_{i}$ be an $\mathfrak{Y}$-module isomorphic to a minimal left ideal of $\mathfrak{B}_{i}$. As an $\mathfrak{U}$-module, we have

$$
\mathfrak{U} \cong t N_{1} \oplus t N_{2} \oplus \cdots \oplus t N_{r} .
$$

Let $M_{1} \# M_{2} \cong \sum_{i} u_{i} N_{i}$ and suppose that $\mathfrak{X} \cong s\left(M_{1} \# M_{2}\right)$ as an $\mathfrak{X}$-module. Then

$$
\sum_{i} t N_{i} \cong \sum_{i} s u_{i} N_{i}
$$

We conclude that the multiplicity $u_{i}$ with which $N_{i}$ appears as a composition factor of $M_{1} \# M_{2}$ is $t / s$. Since the dimension of each $N_{i}$ is $t[D: K]:$ the result follows.

If, in addition to the hypotheses of Theorem 2.4, we also assume that $K$ is perfect, then the proof of Theorem 2.2 of [5] is valid in our more general context and we can express the dimension and multiplicity of each irreducible constituent of $M_{1} \# M_{2}$ as in that theorem. Theorem 2.3 of [5] is valid in our context but is uninteresting. Suppose that $\mathfrak{A}_{1}$ and $\mathfrak{U}_{2}$ are simple algebras over $K$ with centers $L_{1}$ and $L_{2}$ respectively, $L_{1}$ and $L_{2}$ being separable extensions of $K$. Then $\mathfrak{A}_{1} \bigotimes_{K} \mathfrak{A}_{2}$ is simple if and only if $L_{1} \bigotimes_{K} L_{2}$ is a field, and this in turn is equivalent to

$$
\left(L_{1}: K\right)\left(L_{2}: K\right)=\left(L_{1} L_{2}: K\right) \text {. }
$$

We also have

$$
\operatorname{Hom}_{\mathfrak{U}}\left(M_{1} \# M_{2}, M_{1} \# M_{2}\right) \cong \operatorname{Hom}_{\mathfrak{U}_{1}}\left(M_{1} M_{1}\right) \bigotimes_{K} \operatorname{Hom}_{\mathfrak{U}_{2}}\left(M_{2} M_{2}\right) .
$$

Let $D_{i}=\operatorname{Hom}_{\mathfrak{A}_{i}}\left(M_{i}, M_{i}\right)$. Thus $M_{1} \# M_{2}$ is irreducible if and only if $D_{1} \otimes_{K} D_{2}$ is a division algebra. Let $m\left(D_{i}\right)$ denote the index of $D_{i}$. If $K$ is perfect, then $m\left(D_{i}\right)=m_{K}\left(N_{i}\right)$, where $N_{i}$ is an irreducible $\mathfrak{A}_{i}^{E}$ module which is a constituent of $M_{i}^{E}$. Thus, $D_{1} \otimes_{K} D_{2}$ is a division algebra if and only if

$$
m_{K}\left(N_{1}\right) m_{K}\left(N_{2}\right)=m_{K}\left(N_{1} \# N_{2}\right) \text { and }\left(L_{1}: K\right)\left(L_{2}: K\right)=\left(L_{1} L_{2}: K\right) .
$$

In particular, condition (b) of Theorem 2.3 of [5] is unnecessary.

Theorem 2.5 and Corollary 2.5 of [5] are false, as stated. Let $G_{1}$ be the quaternion group of order $8, G_{2}$ the cyclic group of order 3 , and let $K=Q$, the field of rational numbers. Let $\mathfrak{A}_{i}=Q\left(G_{i}\right)$, $i=1,2$. Let $M_{1}$ be the irreducible four-dimensional $Q\left(G_{1}\right)$-module and let $M_{2}$ be the irreducible two-dimensional $Q\left(G_{2}\right)$-module. The conditions of Theorem 2.5 and Corollary 2.6 of [5] are satisfied but $M_{1} \# M_{2}$ is not an irreducible $Q\left(G_{1} \times G_{2}\right)$-module. Using the remarks above and 
the proof of Theorem 2.5 of [5] we can prove the following result.

Proposition 2.5. Let $K$ be perfect and let $\chi_{i}$ be the character of $N_{i}, i=1,2$. If

$$
\left(m_{K}\left(N_{1}\right)\left(K\left(\chi_{1}\right): K\right), m_{K}\left(N_{2}\right)\left(K\left(\chi_{2}\right): K\right)\right)=1,
$$

then $M_{1} \# M_{2}$ is irreducible.

This result generalizes Corollary 1 of $[7, \mathrm{p} .179]$. An analogue for projective representations of Corollary 2.6 of [5] will be proved in $\S 3$.

3. Twisted group algebras. Let $\mathfrak{F}$ be a finite group, $K$ a perfect field. A factor system from $\mathfrak{\mho}$ to $K$ is a function $f$ from $\mathfrak{F} \times \mathfrak{F}$ to the multiplicative group of $K$ such that $f(1,1)=1$, and

$$
f(a, b) f(a b, c)=f(a, b c) f(b, c), \quad \text { for all } a, b, c \in \mathfrak{F} .
$$

The twisted group algebra $K(\mathfrak{F})_{f}$ is defined to be the algebra over $K$ with basis $\{(g)\}_{g \in \mathfrak{F}}$ and multiplication defined by $(a)(b)=f(a, b)(a b)$ for all $a, b \in \mathfrak{F}$. We get the ordinary group algebra when $f(a, b)=1$ for all $a, b \in \mathfrak{F}$. Projective representations of $\mathfrak{F}$ with factor system $f$ correspond to representations of $K(\mathfrak{F})_{f}$.

In discussing splitting field questions for projective representations it seems to be necessary to restrict oneself to one fixed factor system. For example, if $\mathfrak{F}$ is cyclic of order two, $\mathfrak{F}=\langle a\rangle$, then for each integer $n$, other than zero, there exists a factor system of $\mathfrak{F}$ with $f(a, a)=n$. Thus there does not exist any algebraic number field in which every irreducible projective representation of $\mathfrak{F}$ is absolutely irreducible. However, if one works with a fixed factor system $f$, the results of $\S 1$ yield an adequate splitting field theory.

We show that Theorem 2.4 is not true without the restrictions on the normality of the centers of $\mathfrak{A}_{1}$ and $\mathfrak{A}_{2}$. Let $\mathfrak{F}$ be cyclic of order $3, \mathfrak{F}=\langle b\rangle$. Let $f$ be the rational factor system defined by

$$
\begin{gathered}
f(b, b)=f(1, b)=f(b, 1)=f\left(1, b^{2}\right)=f\left(b^{2}, 1\right)=f(1,1)=1, \\
f\left(b^{2}, b\right)=f\left(b, b^{2}\right)=f\left(b^{2}, b^{2}\right)=2 .
\end{gathered}
$$

$Q(\mathfrak{F})_{f} \cong Q(\sqrt[3]{2})$. Thus $Q(\mathfrak{F})_{f}$ has an irreducible 3-dimensional rational module $M$. Let $\mathfrak{A}_{1}=\mathfrak{A}_{2}=Q(\mathfrak{F})_{f}$. Since $Q(\sqrt[3]{2}) \otimes_{Q} Q(\sqrt[3]{2})$ is the sum of all inequivalent $Q$-composites of $Q(\sqrt[3]{2})$ with itself [8, Th. 21, p. 84], we see that

$$
Q(\sqrt[3]{2}) \otimes_{Q} Q(\sqrt[3]{2}) \cong Q(\sqrt[3]{2}) \oplus Q(\omega, \sqrt[3]{2}),
$$


$\omega$ a primitive cube root of 1 . Thus $M \# M$ is the direct sum of a three dimensional and a six dimensional irreducible $Q(\mathfrak{F})_{f} \otimes_{Q} Q(\mathfrak{F})_{f^{-}}$ module.

In view of the above example, we need conditions on $K$ and $f$ to insure that $K(\chi)$ is normal over $K$, where $\chi$ is a character. Let $E$ be a splitting field for $K(\mathfrak{F})_{f}$. $f$ may be viewed as a factor system in $E$ and $E \otimes_{K} K(\mathfrak{F})_{f} \cong E(\mathfrak{F})_{f}$. Let $M$ be an irreducible $K(\mathfrak{F})_{f}$-module and let $\mathfrak{B}$ be the simple component of $K(\mathfrak{F})_{f} / \operatorname{rad}\left(K(\mathfrak{F})_{f}\right.$ such that $M$ is isomorphic to a minimal left ideal of $\mathfrak{B}$. Let $N$ be an irreducible $E(\mathfrak{F})_{f}$-module which is a constituent of $M^{E}$. Let $\chi$ be the character of $N$. The center of $\mathfrak{B}$ is isomorphic to $K(\chi)$, the field generated over $K$ by the values $\{\chi((g))\}_{g \in \mathfrak{F}}$.

Let $g \in \widetilde{F}$ and set $\lambda(g)=\prod_{i=1}^{t} f\left(g, g^{i}\right)$, where $t=|g|$ is the order of $g$. Let $T$ be the representation of $E(\mathfrak{F})_{f}$ afforded by $N$. Then

$$
[T((g))]^{t}=\lambda(g) T(1)=\lambda(g) I,
$$

$I$ the identity matrix of degree $r, r=$ degree $T$, so $\chi(g)$ is the sum of $|g|$-th roots of $\lambda(g)$. If $m$ is the exponent of $₹$ and $K$ contains all $m$-th roots of unity, the $K(\chi)$ is a subfield of an abelian extension of $K$ and so $K(\chi)$ is normal over $K$ with abelian Galois group. If the characteristic of $K$ does not divide $m, K(\chi)$ is a contained in a Kummer extension of $K$.

If $f(x, y)$ is an $v$-th root of unity for all $x, y \in \mathfrak{F}$, then $K(\chi)$ is a subfield of $K(\sqrt[s]{1})$, where $s=m v$. Again we have that $K(\chi)$ is a normal extension of $K$ with abelian Galois group. We have proved:

Proposition 3.1. Assume that one of the following holds:

(a) $K$ contains all $m$-th roots of unity, $m$ the exponent of $\mathfrak{F}$.

(b) $f(x, y)$ is a root of unity for all $x, y \in \mathfrak{F}$.

Then the center of $\mathfrak{B}$ is a finite normal extension of $K$ with abelian Galois group.

REMARKS 1. If $\mathfrak{F}$ is abelian of exponent $m$, the characteristic of $K$ not dividing $m$, and $K$ contains a primitive $m$-th root of unity, then the center of $K(\mathfrak{F})_{f}$ is determined in $[15, \S 6]$. In this case the center of $\mathfrak{B}$ is a Kummer extension of $K$.

2. Suppose that $K$ is an arbitrary field of characteristic $p, p>0$, and $f$ is a factor system of $₹$ in $K$ such that $f(x, y)$ is a root of unity in $K$ for all $x, y \in \mathfrak{F}$. Let $E$ be an algebraic closure of $K$ and let $N$ be an irreducible representation of $E(\widetilde{F})_{f}$ with character $\chi$. Then $K(\chi)$ is a normal extension of $K$. The proofs in $\S 1$ of [5] now carry over unchanged and we can conclude that $m_{K}(N)=1$.

3. Using Proposition 2.5 and Theorem 1.2 we can prove an an- 
alogue of Corollary 2.6 of [5]. Let $\mathfrak{F}_{1}$ and $\mathfrak{F}_{2}$ be finite groups of relatively prime order and let $K$ be a finite field or a field of characteristic zero. Let $\mathfrak{A}_{i}$ be a twisted group algebra of $\widetilde{\mho}_{i}$ over $K$ and let $M_{i}$ be an irreducible $\mathfrak{A}_{i}$-module, $i=1,2$. Let $\mathfrak{B}_{i}$ denote the simple component of $\overline{\mathfrak{T}}_{i}$ such that $M_{i}$ is isomorphic to a minimal left ideal of $\mathfrak{B}_{i}, \mathrm{i}=1,2$. If $\mathfrak{B}_{i}$ is central, $i=1,2$, then $M_{1} \# M_{2}$ is irreducible. The result is immediate from Theorem 1.2(c) and Proposition 2.5 if $K$ is a finite field. Suppose that $K$ has characteristic zero and let $E$ be a splitting field for $\mathfrak{U}_{1}, \mathfrak{A}_{2}$, and $\mathfrak{U}=\mathfrak{A}_{1} \bigotimes_{K} \mathfrak{A}_{2}$. Let $N_{i}$ be an irreducible $\mathfrak{A}_{i}$-module, $i=1,2$, such that $N_{i}$ is a constituent of $M_{i}^{E}$. Then $m_{K}\left(N_{i}\right)$ divides $\left(N_{i}: E\right), i=1,2$, by Theorem 1.2(d). But $\left(N_{i}: E\right)$ divides the order of $\mathfrak{\mho}_{i}, i=1,2\left[4\right.$, Th. 53.16] and so $\left(m_{K}\left(N_{1}\right), m_{K}\left(N_{2}\right)\right)=1$.

4. The Schur index of a projective representation. Let $\mathfrak{F}$ be a finite group and let $K$ be a field. By a linear representation of $\widetilde{F}$ in $K$ we shall mean a homomorphism of $\Im$ into $\mathrm{GL}(r, K)$ for some $r$. A projective representation of $\mathfrak{F}$ in $K$ is a map $T$ from $\mathfrak{F}$ into $\mathrm{GL}(r, K)$ for some $r$, such that $T(x) T(y)=f(x, y) T(x y), T(1)=1_{r}$, where $f$ is a factor system of $\mathfrak{F}$. Let $B$ an algebraically closed field. Let $T$ be an irreducible projective representation of $\mathfrak{F}$ in $B$ with factor system $f$. Let $r$ be the degree of $T$ and let $W$ be a projective representation of $\mathfrak{F}$ in $B$ of degree $r$ with factor system $h . W$ is said to be projectively equivalent to $T$ in $B$ if there exists $A \in \mathrm{GL}(r, B)$ and a function $\rho$ from $\mathfrak{F}$ to $B^{*}$, the multiplicative group of $B$, such that

$$
A^{-1} W(x) A=\rho(x) T(x) \quad \text { for all } \quad x \in \Im \text {. }
$$

If there is a function $\pi$ from $\mathfrak{F}$ to $B^{*}$ such that

$$
f(a, b)=\pi(a) \pi(b) \pi(a b)^{-1} h(a, b) \quad \text { for all } a, b \in \mathfrak{F},
$$

we say that $f$ and $h$ are equivalent. Equivalent projective representations have equivalent factor systems and equivalent factor systems lie in the same equivalence class in $H^{2}\left(\mathfrak{F}, B^{*}\right)$ (trivial action). If the values of $f(a, b), h(a, b), \pi(a)$ and $\pi(b)$, lie in a subfield $K$ of $B$ for all a, $b \in \Re$, we say that $f$ is equivalent to $h$ in $K$. Let the equivalence class of $f$ have order $k$ in $H^{2}\left(\Im, B^{*}\right)$. If the values of $f$ are $k$-th roots of unity we say that $f$ is a normalized factor system. Every projective representation of $₹$ is projectively equivalent to one with a normalized factor system [4, p. 360].

Let $S$ be a projective representation of $\widetilde{\mho}$ in $B$ of degree $r$. We say that $S$ is linearly equivalent to $T$ in $B$ if there exists $D \in \operatorname{GL}(r, B)$ such that

$$
D^{-1} T(x) D=S(x) \quad \text { for all } \quad x \in \mathfrak{F}
$$


Linearly equivalent projective representations have identical factor systems.

Let $F$ be a subfield of $B$ such that $f(x, y) \in F$ for all $\mathrm{x}, y \in \mathfrak{F}$. We say that $T$ is realizable in $F$ if there exists a linearly equivalent projective representation $S$ of $\mathfrak{F}$ in $B$ such that the entries of $S(x)$ lie in $F$ for all $x \in \mathfrak{F}$. Let $\chi(g)=$ trace $T(g), g \in \mathfrak{F}$. In particular, if $T$ is realizable in $F$, then $F$ contains the values $\chi(g), g \in \mathfrak{F}$, as well as the values $f(x, y), x, y \in \mathfrak{F}$. Let $K$ be a subfield of $B$. We define the projective Schur index $m_{K}(T)$ of $T$ over $K$ to be minimum ( $F$ : $K(f, \chi))$, where $K(f, x)$ is the field generated over $K$ by the values $f(x, y), \chi(g)$ for all $x, y, g \in \mathfrak{F}$, the minimum being taken over all fields $F, F \supseteqq K(f, \chi)$, in which $T$ is realizable. We note that if $T$ is a linear representation of $\mathfrak{F}$, then $m_{K}(T)$ is just the usual Schur index.

Let $L$ denote the field $K(f, \chi)$. Let $\mathfrak{A}$ be a simple component of $L(\mathfrak{F})_{f} / \operatorname{rad}\left(L(\mathfrak{F})_{f}\right)$ such that $T$ is afforded by a minimal left ideal of $B \otimes_{L} \mathfrak{A}$. Assume that $L$ is perfect. Applying the results of $\S 1$ to $L(\mathfrak{F})_{f}$ we see that $\mathfrak{A}$ is central simple and $m_{K}(T)=m(D)$, where $m(D)$ is the index of the division algebra component $D$ of $\mathfrak{A}$.

We will be interested in the case when the values of $f$ are roots of unity. If $B$ has characteristic different from zero, it follows from the second remark after Proposition 3.1 that $m_{K}(T)=1$. For this reason we restrict our attention to fields of characteristic zero. Throughout the rest of this paper $B$ will be assumed to be an algebraically closed field of characteristic zero. We note that if $K$ is any subfield of $B$ and $f$ is any factor system of $\mathfrak{F}$ in $K$, then $K(\mathfrak{F})_{f}$ is semi-simple [15, Th. 4.1].

Let $T$ be an irreducible projective representation of $\mathfrak{F}$ in $B$ with factor system $f$. Let $\mathfrak{B}$ be the character group of the multiplicative cyclic group generated by $f$. Following Reynolds [12], we construct an $f$-covering group $\mathfrak{F}^{*}$ of $\mathfrak{F}$ as follows: For $x, y \in \mathfrak{F}$ let $b_{x, y} \in \mathfrak{B}$ be the character such that $b_{x, y}\left(f^{i}\right)=f(x, y)^{i}$. Let $\mathfrak{F}^{*}$ be the set of ordered pairs $(b, x), b \in \mathfrak{B}, x \in \mathfrak{F}$, with multiplication defined by

$$
(b, x)(c, y)=\left(b c b_{x, y}, x y\right) \text {. }
$$

Let $\mathfrak{B}^{*}$ consist of the pairs $(b, 1)$. Then $\mathfrak{F} \cong \mathfrak{F}^{*} / \mathfrak{B}^{*}$. We define a linear representation $T^{*}$ of $\mathfrak{F}^{*}$ by

$$
T^{*}(b, x)=b(f) T(x) \text {. }
$$

We say that $T^{*}$ linearizes $T$.

Proposition 4.1. Let the values of $f$ be roots of unity and let $T, f, T^{*}, \Im^{*}$, be as above. Let $K$ be an arbitrary subfield of the complex numbers. Then $m_{K}(T)=m_{K}\left(T^{*}\right)$. 
Proof. Let $\psi, \chi$ be the characters of $T^{*}, T$ respectively. Then $\psi(b, x)=b(f) \chi(x)$ for all $x \in \mathfrak{F}$. Thus $K(f, \chi)=K(\psi)$. Clearly $T^{*}$ is realizable in a field $F, F \supset K(\psi)$ if and only if $T$ is realizable there. This proves the assertion.

It is frequently possible to determine the projective Schur indices from the properties of $\mathfrak{F}^{*}$. For example, suppose that $\mathfrak{F}$ is a nilpotent group of odd (even) order and the values of $f$ are $v$-th roots of unity, where $v$ is odd (possibly even). Then $\mathfrak{F}^{*}$ is nilpotent of odd (even) order and so $m_{F}(T)=1$ (1 or possibly 2$)[13],[14$, p. 161].

Proposition 4.2. Let $\mathfrak{F}$ have exponent $m$ and let $f$ be a normalized factor system of order $k$ in $H^{2}\left(\mathfrak{F}, B^{*}\right)$. Let $t=k m$ and let $E=$ $Q(\sqrt[t]{1})$. Then $E(\mathfrak{F})_{f}$ is a direct sum of complete matrix rings over $E$.

Proof. Let $S$ be an irreducible complex projective representation of $\mathfrak{F}$ with factor system $f$. It is sufficient to prove that $S$ is realizable in $E$. Let $\mathfrak{F}^{*}$ be the $f$-covering group of $\mathfrak{F}$, and let $S^{*}$ be the linearization of $S$ to $\mathfrak{F}^{*}$. Then $S^{*}$ is realizable in $Q(\sqrt[3]{1}), s$ the exponent of $\mathfrak{F}^{*}$ [4, Th. 41.1]. But $s$ divides $\mathrm{km}$ and so $S$ is realizable in $E$.

A theorem of Kuo [9, Th. 5.1] asserts that, with the notation as above, $\mathrm{km}$ divides the order $n$ of $\mathfrak{F}$. This shows that the field obtained in Proposition 4.2 is a subfield of $Q(\sqrt[n]{1})$. Kuo's proof of her result uses the cohomology theory of groups to reduce to the case when $\mathfrak{F}$ is a $p$-group [1, Th. 10.1, p. 259], and then uses further cohomological information to prove the result in this case. We note that when $\mathfrak{F}$ is a $p$-group this result can also be proved by character theory. If $\mathfrak{F}$ is a finite $p$-group, then so also is $H^{2}\left(\mathfrak{F}, B^{*}\right)[4, \mathrm{Th}$. 53.3].

Proposition 4.3. (Kuo). Let $\mathfrak{F}$ be a finite group of order $p^{t}$ and exponent $p^{s}$. Let $p^{r}$ be the exponent of $H^{2}\left(\mathfrak{F}, B^{*}\right)$. Then $p^{r+s}$ divides $p^{t}$.

Proof. Let $f$ be a factor system of $\mathfrak{F}$ in $B$ of order $p^{r}$ in $H^{2}\left(\mathfrak{F}, B^{*}\right)$. Let $\mathfrak{F}^{*}$ be an $f$-covering group of $\mathfrak{F}$ as defined in Proposition 4.1. Then $\mathfrak{F}^{*}$ has a central subgroup $\mathfrak{S}$ of order $p^{r}$ and $\mathfrak{F}^{*} / \mathfrak{S} \cong \mathfrak{F}$ has a cyclic subgroup of order $p^{s}$. Thus $\mathfrak{F}^{*}$ has an abelian subgroup $\mathfrak{M}$ of order $p^{u}, u=r+s$. It is sufficient to prove that $p^{r} \leqq\left[\mathfrak{F}^{*}: \mathfrak{M}\right]$. By Frobenius reciprocity it is sufficient to prove that $\mathfrak{F}^{*}$ has an absolutely irreducible representation of degree greater than 
or equal to $p^{r}[4$, Exercise 8, p. 279]. Let $S$ be an irreducible projective representation of $\mathfrak{F}$ in $B$ with factor system $f$ (afforded by an irreducible $B(\mathfrak{F})_{f}$-module). Let $p^{v}$ be the degree of $S$. Taking determinants in the equation

$$
S(x) S(y)=f(x, y) S(x y), \quad x, y \in \mathfrak{F}
$$

we see that the order $p^{r}$ of $f$ in $H^{2}\left(\mathfrak{F}, B^{*}\right)$ divides $p^{v}$. Let $S^{*}$ be a linearization of $S$ to $\mathfrak{F}^{*}$. Since $S^{*}$ is an absolutely irreducible representation of $\mathfrak{F}^{*}$ of degree greater than or equal to $p^{r}$, we are done.

Proposition 4.2 is false if $E$ is replaced by $Q(\sqrt[m]{1})$. The simplest example of this is when $\mathfrak{F}=Z_{2} \times Z_{2}$ and $Q(\mathfrak{F})_{f}$ is the rational quaternion algebra, $Q$ the field of rationals. We note also that, in view of Kuo's theorem, Proposition 4.2 is a strengthening of a theorem of Reynolds [12, Th. 5].

We consider next the question of how the Schur index behaves under projective equivalence. It is possible for $T$ and $S$ to be projectively equivalent irreducible projective representations of $\mathfrak{F}$, both having normalized factor systems, but having different Schur indices over the rationals. Let $\mathfrak{F}=Z_{2} \times Z_{2}$ and let $f, g$ be normalized factor systems of $\mathfrak{F}$ so that $Q(\mathfrak{F})_{f} \cong(Q)_{2}$, the algebra of $2 \times 2$ matrices over the rationals $Q$, while $Q(\mathfrak{F})_{g} \cong Q^{\prime}$, the rational Quaternion division algebra.

$$
B(\mathfrak{F})_{f} \cong B(\mathfrak{F})_{g} \cong(B)_{2} .
$$

Let $T, S$ be irreducible projective representations of $\mathfrak{F}$ in $B$ with factor systems $f, g$ respectively. Then $m_{Q}(T)=1$, while $m_{Q}(S)=2$. The $f, g$-covering groups of $\mathfrak{F}$ are respectively the dihedral and quaternion groups of order 8 .

Suppose that $T$ and $S$ are projectively equivalent irreducible projective representations of $\mathfrak{F}$ in $B$ with associated factor systems $f$ and $g$ respectively. The possible values of the Schur index over $Q$ are bounded; by Theorem $1.2(\mathrm{~b})$ we have $m_{Q}(T) \leqq \sqrt{n}$, where $n$ is the order of $\mathfrak{F}$. Within these bounds we can have various possibilities for $m_{Q}(S)$. We show next that $S$ can always be found such that $m_{Q}(S)=1$.

Proposition 4.4. Let $T$ be an irreducible projective representation in $B$ of the finite group $\mathfrak{F}$. Then $T$ is projectively equivalent in $B$ to a projective representation $S$ of $\mathfrak{F}$ such that $m_{Q}(S)=1$. $\quad S$ may be chosen to have a factor system whose values are $|\mathfrak{F}|$-th roots of unity. 
Proof. Let $f$ be the factor system associated with $T$ and let $\chi$ be the projective character of $\mathfrak{F}$ afforded by $T$.

$$
m_{Q}(T)=\operatorname{minimum}(F: Q(f, \chi)),
$$

the minimum being taken over all fields $F$ in which $T$ is realizable. We will construct $S$ by suitably enlarging $Q(f, \chi)$. We may clearly assume that $f$ is normalized. If $|\mathfrak{F}|<3$, the result is trivial since $S$ may be taken to be an ordinary representation. Let $x$ and $y$ be nonidentity elements of $\mathfrak{F}$ with $x \neq y$. Let $\zeta$ be a primitive $|\mathfrak{F}|$-th root of unity and define a function $\rho$ from $\mathfrak{F}$ to $B^{*}$ by $\rho(a)=1$, $a \neq x$, and $\rho(x)=\zeta f(x, y)^{-1}$. Let $S(a)=\rho(a) T(a)$ for all $a \in \mathfrak{F}$. Then $S$ is projectively equivalent to $T$. Let $g$ be the factor system associated with $S$. Then $g(x, y)=\zeta$. Let $E=Q(\zeta)$. By Proposition 4.2, $E(\mathfrak{F})_{f}$ is the direct sum of complete matrix rings over $E$. By Theorem 1.2(b), $T$ is realizable in $E$ so we may assume that the entries of $T(a)$ lie in $E$ for all $a \in \mathfrak{F}$. We see that $S$ is realizable in $E$. Let $\psi$ be the projective character of $\widetilde{F}$ afforded by $S$. It remains to show that $Q(g, \psi)=E$. However, this follows immediately from $g(x, y)=\zeta$. The factor system $g$ of $S$ have values which are $|\mathfrak{F}|$-th roots of unity.

The author wishes to thank the referee for his many helpful suggestions.

\section{REFERENCES}

1. R. Brauer, Über systeme hyperkomplexer Zahlen, Math. Zeit. 30 (1929), 79-107.

2. - Untersuchungen über die arithmetischen eigenschaften von gruppen linearer substitutionen II, Math. Zeit. 31 (1930), 737-747.

3. H. Cartan and S. Eilenberg, Homological algebra, Princeton University Press, Princeton, 1956.

4. C. W. Curtis and I. Reiner, Representation theory of finite groups and associative Algebras, Interscience, New York, 1962.

5. B. Fein, Representations of direct products of finite groups, Pacific J. Math. 20 (1967), 45-58.

6. J. Ivan, The radical and semi-simplicity of direct products of direct products of algebras, (Slovak), Mat.-Fyz. Časopis. Slovensk. Akad. Vied. 7 (1957), 158-167.

7. N. Jacobson, The Structure of rings, Amer. Math, Soc., Providence, 1956.

8. - Lectures in abstract algebra, Vol. III, Van Nostrand, Princeton, 1964.

9. T. Kuo, On the exponent of $H^{n}(G, Z)$, J. Algebra 7 (1967), 160-167.

10. E. Noether, Nichtkommutative algebra, Math. Zeit. 37 (1933), 513-541.

11. I. Reiner, The Schur index in the theory of group representations, Michigan Math. J. 8 (1961), 39-47.

12. W. F. Reynolds, Projective representations of finite groups in cyclotomic fields, Illinois, J. Math 9 (1965), 191-198.

13. P. Roquette, Realisierung von Darstellungen endlicher nilpotenter gruppen, Archiv der Math. 9 (1958), 241-250.

14. L. Solomon, The representations of finite groups in algebraic number fields, J. Math. Soc. Japan 13 (1961), 144-164. 
15. K. Yamazaki, On projective representations and ring extensions of finite groups, J. Fac. Sci. University Tokyo, Sect. I, 10 (1964), 147-195.

Received November 9, 1967. The research on this paper was supported in part by NSF Grant GP-5497.

UNIVERSity of CALIFORNIA, LOS ANGELES 


\section{PACIFIC JOURNAL OF MATHEMATICS}

\section{EDITORS}

H. ROYDEN

Stanford University

Stanford, California

R. R Phelps

University of Washington

Seattle, Washington 98105
J. DUGUNDJI

Department of Mathematics

University of Southern California

Los Angeles, California 90007

RICHARD ARENS

University of California

Los Angeles, California 90024

\section{ASSOCIATE EDITORS}
E. F. BECKENBACH
B. H. NeumanN
F. WOLF
K. YosidA

\section{SUPPORTING INSTITUTIONS}

UNIVERSITY OF BRITISH COLUMBIA

CALIFORNIA INSTITUTE OF TECHNOLOGY

UNIVERSITY OF CALIFORNIA

MONTANA STATE UNIVERSITY

UNIVERSITY OF NEVADA

NEW MEXICO STATE UNIVERSITY

OREGON STATE UNIVERSITY

UNIVERSITY OF OREGON

OSAKA UNIVERSITY

UNIVERSITY OF SOUTHERN CALIFORNIA
STANFORD UNIVERSITY

UNIVERSITY OF TOKYO

UNIVERSITY OF UTAH

WASHINGTON STATE UNIVERSITY

UNIVERSITY OF WASHINGTON

AMERICAN MATHEMATICAL SOCIETY CHEVRON RESEARCH CORPORATION TRW SYSTEMS

NAVAL WEAPONS CENTER 


\section{Pacific Journal of Mathematics

Vol. 28, No. $1 \quad$ March, 1969

Patrick Robert Ahern, On the geometry of the unit ball in the space of real annihilating measures .............................. 1

Kirby Alan Baker, Equational classes of modular lattices ............. 9

E. F. Beckenbach and Gerald Andrew Hutchison, Meromorphic minimal surfaces ......................................... 17

Tae Ho Choe, Intrinsic topologies in a topological lattice ..............

John Bligh Conway, A theorem on sequential convergence of measures and

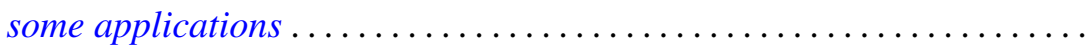

Roger Cuppens, On the decomposition of infinitely divisible probability laws without normal factor.

Lynn Harry Erbe, Nonoscillatory solutions of second order nonlinear

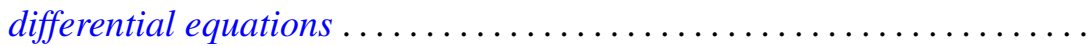

Burton I. Fein, The Schur index for projective representations of finite

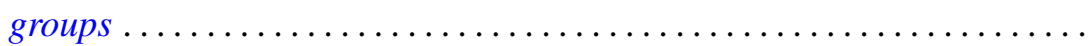

Stanley P. Gudder, A note on proposition observables............... 101

Kenneth Kapp, On Croisot's theory of decompositions ............... 105

Robert P. Kaufman, Gap series and an example to Malliavin's theorem . . . 117

E. J. McShane, Robert Breckenridge Warfield, Jr. and V. M. Warfield,

Invariant extensions of linear functionals, with applications to measures and stochastic processes ................

Marvin Victor Mielke, Rearrangement of spherical modifications ...

Akio Osada, On unicity of capacity functions ..............

Donald Steven Passman, Some 5/2 transitive permutation groups ...

Harold L. Peterson, Jr., Regular and irregular measures on groups and dyadic spaces...

Habib Salehi, On interpolation of $q$-variate stationary stochastic processes...

Michael Samuel Skaff, Vector valued Orlicz spaces generalized

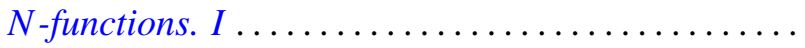

A. J. Ward, On $H$-equivalence of uniformities. II...........

Thomas Paul Whaley, Algebras satisfying the descending chain condition for subalgebras...

G. K. White, On subgroups of fixed index

Martin Michael Zuckerman, A unifying condition for implications among the axioms of choice for finite sets ................. 